\title{
MS41-P06 | DYNAMIC THEORY OF PROTEIN CRYSTALLIZATION
}

Hasek, Jindrich (Institute of Biotechnology, Academy of Sciences, Vestec, CZE); Skálová, Tereza (Institute of Biotechnology, Academy of Sciences, Vestec, CZE); Dušková, Jarmila (Institute of Biotechnology, Academy of Sciences, Vestec, CZE); Koval, Tomáš (Institute of Biotechnology of the Czech Academy of Sciences, Vestec, CZE); Dohnálek, Jan (Institute of Biotechnology of the Czech Academy of Sciences, Vestec, CZE)

Classical crystallization theories use model of ideally dissolved molecules in homogeneous crystallization solution. However, components of crystallization solution often form temporary adducts leading to preorganization of target molecules. Adhesion of all components of crystallization solution, competition of different adhesion modes and formation of temporary protein clusters is of principal importance namely in protein crystallization.

The new dynamic theory of protein crystallization is based on analysis of intermolecular interactions responsible for regular deposition of protein molecules in crystal. It introduces a general term "adhesion mode" describing the tendency of molecules to realize a specific configuration during interaction of two molecules in a given environment. The new concept describing crystallization as a competition between possible adhesion modes mutually incompatible within a given crystal form gives us a rational tool to see why "crysallization attempt" leads to amorphous precipitate or for to regular crystalline form. Crystallographer equipped with knowledge of basic principles of intermolecular adhesion can rationalize any crystallization method respecting the „principle of a single adhesion mode".

Recognition of intermolecular adhesion as a principle driving force of protein crystallization explains many formerly mysterious effects observed in practice in many crystallization techniques. It

- explains excellent performance of PEG, $\left(\mathrm{NH}_{3}\right)_{2} \mathrm{SO}_{4}$, etc in crystallization experiments,

- shows how the composition of mother liqueur influences the crystal form (space group),

- explains initiation of crystallization in under-saturated solution in the confined space of cavities in some nanoporous materials,

- allows preparation of better crystals and different polymorphs of the same protein. 